\title{
Factors Associated With a Negative Emotional Response to News Media and Nationwide Emergency Text Alerts During the COVID-19 Outbreak in Korea
}

\author{
Ju-Yeon Lee ${ }^{1,2}$, Mina Kim², Min Jhon', Ju-Wan Kim¹, \\ Seunghyong Ryu ${ }^{1}$, Jae-Min Kim¹, and Sung-Wan Kim ${ }^{1,2} \bowtie$ \\ 'Department of Psychiatry, Chonnam National University Medical School, Gwangju, Republic of Korea \\ ${ }^{2}$ Gwang-ju Mental Health Commission, Gwangju, Republic of Korea
}

\begin{abstract}
Objective To prevent the spread of infection in Korea during the corona virus disease 2019 (COVID-19) outbreak, real-time warnings have been sent to all residents via mobile phones. This study examined the factors associated with the negative emotional response to media news and emergency text alerts in the COVID-19 pandemic.

Methods A survey was completed by 1,500 adults from an online public panel in three regions. We used Patient Health Questionnaire-9 (PHQ-9), generalized anxiety disorder-7 (GAD-7) and Perceived Stress Scale-10 (PSS-10) to assess the level of depression, anxiety and stress, respectively. Questionnaires related to COVID-19 included fear of infection, and fear of disclose of contact-tracing information.

Results The negative emotional response on both news media information and emergency alert text messages about COVID-19 was associated with fear of COVID-19 infection and high anxiety. The biggest outbreak city, Daegu was associated with the less negative emotional response on emergency alert text messages. Fear of disclose of contact-tracing information was significantly associated with negative emotional perception on emergency alter text messages.
\end{abstract}

Conclusion Our results suggest that effective information providing services with considering vulnerable groups are needed to promote acceptance and eliminate negative emotion for disease related information.

Psychiatry Investig 2021;18(9):825-830

Keywords COVID-19; Media coverage; Emergency text alarm; Anxiety; Stress.

\section{INTRODUCTION}

Since the corona virus disease 2019 (COVID-19) pandemic began in March 2020, most news reports through the mass media and internet have contained information related to this infection. In Korea, the director of the Korean Centers for Disease Control and Prevention has held a daily media briefing regarding the confirmed cases and mortality rate of the disease. The Korean government also sends COVID-19-related emergency alerts simultaneously to all mobile phone users in Korea at city and district levels. These messages include details of re-

Received: March 8, 2021 Revised: May 23, 2021

Accepted: July 1, 2021

$\triangle$ Correspondence: Sung-Wan Kim, MD, PhD

Department of Psychiatry, Chonnam National University Medical School, 160 Baekseo-ro, Dong-gu, Gwangju 61469, Republic of Korea

Tel: +82-62-220-6148, Fax: +82-62-225-2351

E-mail: swkim@chonnam.ac.kr

(a) This is an Open Access article distributed under the terms of the Creative Commons Attribution Non-Commercial License (https://creativecommons.org/licenses/by$\mathrm{nc} / 4.0$ ) which permits unrestricted non-commercial use, distribution, and reproduction in any medium, provided the original work is properly cited. cently confirmed cases and contact-tracing information, including the time and location of infected peoples' visits. ${ }^{1}$ Media reports on an epidemic can provide important information to the public and can induce positive health behavior in individuals. ${ }^{2}$ However, the mass media also transmits the negative impact of disasters, which is a risk factor for emotional problems. ${ }^{3}$

Fear increases in the face of an uncertain or uncontrollable threat and during a health crisis, and public depends on the media to seek out confirmation and make decisions regarding preventative behavior. ${ }^{4.5}$ Previous study suggested the importance of providing timely and accurate information to the public in ensuring that people are able to avoid the risk of infection. ${ }^{6}$ With the development of information and communication technology (ICT) of Korea, citizens have been able to access COVID-19 related information on various channels. However, there is insufficient evidence on factors associated with negative emotional responses to COVID-19-related information received by text alert messages or through the news media. Therefore, this study focused on the traditional news media and national 
emergency alert text messages provided during the COVID-19 outbreak to explore factors associated with negative emotional perception of the information delivery methods. We believe that our study can contribute to developing effective public strategies to reduce negative emotional responses related to exposure to COVID-19 information.

\section{METHODS}

\section{Study design and participants}

This study is a part of a mental health survey of the psychosocial effects of COVID-19 on the general population, to investigate the impact on mental health problems during the COVID-19 pandemic. In this study, we grouped individuals according to their responses to a questionnaire about the degree of immediate negative emotion to COVID-19-related news and emergency text alert messages. The study enrolled 1,500 adults (750 of each sex) between 19 and 65 years of age who resided in Korea. Between April 24 and May 5 (Supplementary Figure 1 in the online-only Data Supplement), 2020 (3 months after the COVID-19 outbreak began, and when 10,694 confirmed cases had occurred), we conducted an anonymous online survey of representative samples of residents from the capital area with 1,286 confirmed cases (12\%), Daegu city with 6,836 confirmed cases (63.9\%), which was the center the Korean COVID-19 outbreak, and Gwangju, the city with the fewest confirmed cases, with 30 confirmed cases $(0.3 \%) .^{7}$ All respondents provided informed consent. The Institutional Review Board of Chonnam National University Hospital approved the study (CNUH2020-092).

\section{Outcome measures}

\section{Sociodemographic characteristics}

Demographic variables used to determine participant characteristics included sex, age, marital status, and residence location.

\section{Negative emotional response to news media and} emergency text alert messages

Two items were used to examine the participants' emotional perception of the news media and emergency text alert messages sent via mobile phones: "I feel fear when I am exposed to news about COVID-19" and "I get anxious when I receive emergency alert text messages about COVID-19 via my mobile phone." All items on the questionnaire were rated using a 5 -point Likert scale, from "not at all" (1 point) to "very much" (5 points). Answering any item with either "much" or "very much" was construed as indicative of the group with a negative emotional response ('yes' group).

\section{COVID-19 related questionnaire}

From a questionnaire that we developed to investigate the psychosocial experiences and distress associated with COVID-19, ${ }^{7}$ we examined the factor 'fear of COVID-19 infection' in this study. The following question about fear of disclosing contact-tracing information was asked: "If I am confirmed to have COVID-19 infection, I am afraid that all my movements will be released." This was also rated using a 5-point Likert scale, from "not at all" (1 point) to "very much" (5 points).

\section{Assessment of psychological symptoms}

The Patient Health Questionnaire-9 (PHQ-9) was used to measure the severity of self-reported depression. ${ }^{8}$ The total score range from 0 to 27, with a higher score indicating greater selfreported depression. Anxiety was measured using the generalized anxiety disorder-7 (GAD-7). ${ }^{9}$ Each item of GAD-7 asks about anxiety symptoms during the preceding two weeks. It is rated on a 4-point scale (from 0 to 3 ) and the total score ranges from 0 to 21, and higher scores reflecting greater anxiety. The Korean version of the GAD-7 has been validated. Stress was measured using the Perceived Stress Scale-10 (PSS-10). ${ }^{10}$ PSS10 consists of 10 items addressing specific feelings experienced in the last month. Respondents were asked to rate each item on a five-point Likert scale with higher scores indicating greater psychological stress.

\section{Statistical analyses}

Between groups comparisons of sociodemographic and clinical characteristics were analyzed using the chi-square test for categorical variables and independent $t$-tests for continuous variables. Logistic regression was used to examine the factors affecting the negative emotional perception of the news media and emergency text alert messages. SPSS for Windows ver. 21.0 (IBM Corp., Armonk, NY, USA) was used to perform the statistical tests. All statistical tests were two-tailed and $\mathrm{p}<0.05$ were considered significant.

\section{RESULTS}

\section{General characteristics and differences in the negative emotional response on news media and emergency text alert message}

The participants' mean age was 40.2 years (males, $40.4 \pm 11.9$ years; females, $40.1 \pm 11.7$ years), and 740 (49.3\%) were married. $43.7 \%$ of the participants reported that they felt fear when they were exposed to the news about COVID-19, whereas 32.9\% of the participants reported that they got to be anxious when they received emergency alert text message about COVID-19 on mobile phone (Table 1).

The negative emotional perception on media news about 
Table 1. Sociodemographic and clinical characteristics according to the negative emotional perception on COVID-19 related information

\begin{tabular}{|c|c|c|c|c|c|c|c|}
\hline \multirow[b]{2}{*}{ Variable } & \multirow[b]{2}{*}{$\begin{array}{l}\mathrm{N}(\%) \text { or } \\
\text { mean }(\mathrm{SD})\end{array}$} & \multicolumn{3}{|c|}{ News media } & \multicolumn{3}{|c|}{ Emergency alert text message } \\
\hline & & $\begin{array}{c}\text { No } \\
(\mathrm{N}=844 \\
[56.3 \%]) \\
\end{array}$ & $\begin{array}{c}\text { Yes } \\
(\mathrm{N}=656 \\
[43.7 \%])\end{array}$ & $\begin{array}{c}\text { t or } \chi^{2} \\
\text { (p-value) }\end{array}$ & $\begin{array}{c}\text { No } \\
(\mathrm{N}=1,007 \\
[67.1 \%]) \\
\end{array}$ & $\begin{array}{c}\text { Yes } \\
(\mathrm{N}=493 \\
[32.9 \%])\end{array}$ & $\begin{array}{c}\text { t or } \chi^{2} \\
\text { (p-value) }\end{array}$ \\
\hline Gender & & & & $27.092(<0.001)$ & & & $21.830(<0.001)$ \\
\hline Male & $750(50)$ & $472(62.9)$ & $278(37.1)$ & & $546(72.8)$ & $204(27.2)$ & \\
\hline Female & $750(50)$ & $372(49.6)$ & $378(50.4)$ & & $461(61.5)$ & $289(38.5)$ & \\
\hline Age (yr) & $40.24(11.88)$ & $39.5 \pm 11.5$ & $41.3 \pm 12.3$ & $-2.924(0.004)$ & $39.9 \pm 11.8$ & $40.9 \pm 12.0$ & $-1.686(0.092)$ \\
\hline Marriage & & & & $7.539(0.006)$ & & & $10.017(0.002)$ \\
\hline Married & $740(49.3)$ & $390(52.7)$ & $350(47.3)$ & & $468(63.2)$ & $272(36.8)$ & \\
\hline Single & $760(50.7)$ & $454(59.7)$ & $306(40.3)$ & & $539(70.9)$ & $221(29.1)$ & \\
\hline Area & & & & $0.883(0.643)$ & & & $19.259(<0.001)$ \\
\hline Capital region & $500(33.3)$ & $273(54.6)$ & $227(45.4)$ & & $309(61.8)$ & $191(38.2)$ & \\
\hline Daegu & $500(33.3)$ & $284(56.8)$ & $216(43.3)$ & & $372(74.4)$ & $128(25.6)$ & \\
\hline Gwangju & $500(33.3)$ & $287(57.4)$ & $213(218.7)$ & & $326(65.2)$ & $174(34.8)$ & \\
\hline Fear of COVID-19 infection & $3.81(0.77)$ & $3.52(0.76)$ & $4.18(0.62)$ & $-18.159(<0.001)$ & $3.62(0.77)$ & $4.18(0.62)$ & $-14.136(<0.001)$ \\
\hline $\begin{array}{l}\text { Fear of disclose of } \\
\text { contact-tracing information }\end{array}$ & $3.35(1.26)$ & $3.09(1.24)$ & $3.69(1.2)$ & $-9.346(<0.001)$ & $3.17(1.25)$ & $3.73(1.187)$ & $-8.327(<0.001)$ \\
\hline PHQ-9 & $5.92(5.23)$ & $5.34(4.94)$ & $6.67(5.49)$ & $-4.899(<0.001)$ & $5.30(4.83)$ & $7.19(5.75)$ & $-6.651(<0.001)$ \\
\hline GAD-7 & $4.37(4.51)$ & $3.53(4.17)$ & $5.22(4.76)$ & $-7.331(<0.001)$ & $3.49(3.98)$ & $5.86(5.09)$ & $-9.836(<0.001)$ \\
\hline PSS-10 & $18.97(4.83)$ & $18.08(4.84)$ & $20.12(4.56)$ & $-8.275(<0.001)$ & $18.35(4.81)$ & $20.26(4.60)$ & $-7.319(<0.001)$ \\
\hline
\end{tabular}

PHQ-9, Patient Health Questionnaire-9; GAD-7, generalized anxiety disorder; PSS-10, Perceived Stress Scale-10; SD, standard deviation

COVID-19 was more likely to be in female, old age, married, people having fear of COVID-19 infection and fear of disclose of contact-tracing information. The people with negative emotional perception on news about COVID-19 reported greater depression, anxiety symptom and psychological stress than those without negative emotional perception. Anxious response on emergency alert text message about COVID-19 was more likely to be in female, married, and people having fear of COVID-19 infection and fear of disclose of contact-tracing information and less likely to be in people lived in Daegu city (pandemic area). The people with anxious response on emergency alert text message about COVID-19 reported greater depression, general anxiety symptom and psychological stress than those without anxious response.

\section{Multivariate analysis of factors associated with negative emotional response}

In the multivariate logistic regression analyses (Table 2), negative emotional perception of news about COVID-19 information was significantly associated with female, fear of COVID-19 infection, high anxiety, and psychological stress. An anxious response to emergency alert text messages about COVID-19 was significantly associated with female, married, fear of COVID-19 infection, and high anxiety. The biggest outbreak city, Daegu, was negatively associated with an anxious response to emer- gency alert text messages $(\mathrm{OR}=0.475, \mathrm{p}<0.001)$. Fear of disclosure of contact-tracing information was significantly associated with a negative emotional response to emergency alert text messages about COVID-19.

\section{DISCUSSION}

In this study, it was found that negative emotional perception on news media (43.7\%) that can be voluntarily accessed was relatively greater than emergency alert message which is almost compulsory and sudden (32.9\%). While information via news coverages may involve subjective interpretation and carry a considerable amount of aversive images, emergency alert text message is focused on conveying identified facts, tracing information of confirmed case, which can help citizens decide their actions right away. Our results suggest that citizens exposed to pandemic situation may feel more helpful with accurate and prompt information via alert message.

In this study, fear of COVID-19 infection was significantly associated with negative emotional perceptions of both types of online media. The COVID-19 pandemic has been accompanied by the "infodemic," which refers to the perils of excessive information in relation to COVID-19, including misinformation and fake news through social media. ${ }^{11}$ Media reporting can produce secondary trauma and has been criticized for cre- 
Table 2. Multivariate associations of participant characteristics with negative emotional perception of COVID-19-related information

\begin{tabular}{|c|c|c|c|c|}
\hline & \multicolumn{2}{|c|}{ News media } & \multicolumn{2}{|c|}{ Emergency alert text messages } \\
\hline & OR $(95 \% \mathrm{CI})$ & $\mathrm{p}$ & OR (95\% CI) & $\mathrm{p}$ \\
\hline Sex, female & $1.454(1.151-1.837)$ & 0.002 & $1.460(1.143-1.865)$ & 0.002 \\
\hline Age & $1.010(0.998-1.023)$ & 0.100 & $1.001(0.988-1.014)$ & 0.878 \\
\hline Marriage, married & $1.109(0.826-1.489)$ & 0.492 & $1.460(1.072-1.987)$ & 0.016 \\
\hline \multicolumn{5}{|l|}{ Area } \\
\hline Capital region & Reference & & Reference & \\
\hline Daegu & $0.836(0.630-1.110)$ & 0.216 & $0.475(0.352-0.641)$ & $<0.001$ \\
\hline Gwangju & $0.975(0.735-1.293)$ & 0.858 & $0.934(0.700-1.245)$ & 0.641 \\
\hline Fear of COVID-19 infection & $3.444(2.823-4.202)$ & $<0.001$ & $2.713(2.212-3.328)$ & $<0.001$ \\
\hline Fear of disclose of contact-tracing information & $1.102(0.995-1.220)$ & 0.061 & $1.116(1.003-1.241)$ & 0.044 \\
\hline PHQ-9 & $0.988(0.953-1.024)$ & 0.512 & $0.989(0.954-1.026)$ & 0.555 \\
\hline GAD-7 & $1.050(1.008-1.094)$ & 0.020 & $1.107(1.062-1.155)$ & $<0.001$ \\
\hline PSS-10 & $1.032(1.001-1.064)$ & 0.040 & $1.008(0.978-1.040)$ & 0.598 \\
\hline
\end{tabular}

PHQ-9, Patient Health Questionnaire-9; GAD-7, generalized anxiety disorder; PSS-10, Perceived Stress Scale-10; OR, odds ratio; CI, confidence interval

ating public fear in the face of emerging health threats. ${ }^{12}$ The results of our study imply that the media needs to reduce excessive fear by providing information that is correct and can aid citizens' comprehension. Fear of the disclosure of contact-tracing information was significantly associated with a negative emotional response only for emergency alert text messages about COVID-19. Many have pointed out that the disclosure of information is a main component that contributed to the rapid spread of infectious disease. ${ }^{13}$ Information spread through mass media is important for physical distancing, which is one of the most effective strategies for preventing the spread of this virus. However, as excessive disclosure of personal information may cause negative emotions in citizens, it is necessary to share information carefully, considering the pros and cons of information disclosure.

Notably, Daegu, the city with the biggest outbreak, was associated with less fearful perception of COVID-19 information via emergency text messages in this study. The fear response of Daegu citizens to alert message may have been reduced by excessive COVID-19 related information in the face of a surge in new confirmed cases and exposure to a continuous threat. A similar phenomenon has been described as 'media fatigue."14 It has been shown that individuals may be more likely to take precautions against disease with the initial reports of an epidemic, but individual sensitivity and emotional responsiveness may diminish after repeated media exposure. ${ }^{15,16}$ Our results suggest that collective emotional insensitivity may occur in the epicenter of an infectious disease outbreak compared to other areas with fewer confirmed cases.

We found that high reporting of anxiety and stress was associated with a negative emotional perception of COVID-19 in- formation. Preexisting mental health conditions can predict the reaction to media reports. ${ }^{17}$ Anxiety symptoms result in distorted information processing, leading to perceptual bias. ${ }^{18}$ Our result suggests that anxiety may have significant impact on emotional interpretation when people exposed to media, which may provoke fear. Furthermore, high stress and anxiety levels are associated with maladaptive coping strategies, such as avoidance and rumination in stressful situations..$^{19}$ High levels of stress are associated with maladaptive coping strategies such as avoidance and rumination. ${ }^{19-21}$ It is possible that this maladaptive coping under high stressful condition may serve to overly focus on the signs of threat and rise negative emotion. Our results suggest that improving individuals' ability to regulate emotions and effective strategies for coping with stress are needed to increase comfortable acceptance of COVID-19 information and to take proven precautionary measures. It is also recommended that people with high anxiety and distress reduce the time they spend searching for news.

With regard to sociodemographic factors, we found that female was associated with the perception of fear on both news and emergency alert message about COVID-19 information. This is in line with previous studies, indicating that after traumatic events, women reported greater trauma-related fear than men. ${ }^{22,23}$ Recent studies conducted during COVID-19 pandemic also reported that women had more psychological symptoms including anxiety and post-traumatic stress than males..$^{24,25}$ We found that marital status was associated with the negative emotional perception on emergency text message. It is possible that there were many other family members besides themselves who could be a potential case for COVID-19 infection, so they might have to deal with the fears of shame, blame, and 
stigma caused by uncontrollable family member's contact with virus whenever they received the alert text.

Some potential limitations should be noted in this study. First, our cross-sectional study design did not allow us to establish a causal relationship between psychosocial factors and the responses of participants for information exposure. In addition, we did not distinguish between the immediate emotional response to the alert messages and the underlying/chronic emotional status related to COVID-19. Therefore, longitudinal studies and additional analyses are needed. Second, the lack of structured interviews to assess psychological status may have confounded our results. Third, as the survey was conducted online, which is suitable for rapid assessment, so some respondent bias, such as elder citizens, may have affected the results.

In conclusion, our findings showed that COVID-19 related fear of including privacy exposure as well as infection could increase the negative emotional response on online media. In addition, individuals with anxiety and stress were vulnerable to negative emotion for COVID-19 information. During an ongoing threat from a novel disease and the long-lasting $\mathrm{CO}$ VID-19 pandemic, we need to establish effective communication measures that will reduce the public's fears and increase preventive healthy behaviour.

\section{Supplementary Materials}

The online-only Data Supplement is available with this article at https://doi.org/10.30773/pi.2021.0087.

\section{Availability of Data and Material}

The datasets generated or analyzed during the study are available from the corresponding author on reasonable request.

\section{Conflicts of Interest}

The authors have no potential conflicts of interest to disclose.

\section{Author Contributions}

Conceptualization: Sung-Wan Kim, Ju-Yeon Lee. Formal analysis: SungWan Kim, Mina Kim, Ju-Yeon Lee. Funding acquisition: Sung-Wan Kim. Investigation: all authors. Methodology: all authors. Project administration: Sung-Wan Kim, Ju-Yeon Lee. Software: Mina Kim, Min Jhon. Supervision: Jae-Min Kim, Sung-Wan Kim. Validation: all authors. Visualization: all authors. Writing_-original draft: Ju-Yeon Lee. Writing_review \& editing: Jae-Min Kim, Sung-Wan Kim.

\section{ORCID iDs}

Ju-Yeon Lee

Mina Kim

Min Jhon

Ju-Wan Kim

Seunghyong Ryu

Jae-Min Kim

Sung-Wan Kim https://orcid.org/0000-0003-0653-7223 https://orcid.org/0000-0003-2295-6786 https://orcid.org/0000-0002-0408-768X https://orcid.org/0000-0002-9888-1090 https://orcid.org/0000-0001-6127-760X https://orcid.org/0000-0001-7409-6306 https://orcid.org/0000-0002-6739-2163

\section{Funding Statement}

This research was supported by a grant of Patient-Centered Clinical Research Coordinating Center (PACEN) funded by the Ministry of Health \&
Welfare, Republic of Korea (grant number : HI19C0481, HC19C0316).

\section{REFERENCES}

1. Park S, Choi GJ, Ko H. Information technology-based tracing strategy in response to COVID-19 in South Korea-Privacy Controversies. JAMA 2020;323:2129-2130.

2. Majumder MS, Kluberg S, Santillana M, Mekaru S, Brownstein JS. 2014 Ebola Outbreak: Media events track changes in observed reprodutive number. PLoS Curr 2015;7:ecurrents.outbreaks.e6659013c1d7f11bdab 6a20705d1e865.

3. Gao J, Zheng P, Jia Y, Chen H, Mao Y, Chen S, et al. Mental health problems and social media exposure during COVID-19 outbreak. PLoS One 2020;15:e0231924.

4. Lerner JS, Keltner D. Beyond valence: toward a model of emotion-specific influences on judgement and choice. Cogn Emot 2000;14:473-493.

5. Lerner JS, Keltner D. Fear, anger, and risk. J Pers Soc Psychol 2001;81: 146-159.

6. Noh JW, Yoo KB, Kwon YD, Hong JH, Lee Y, Park K. Effect of information disclosure policy on control of infectious disease: MERS-CoV outbreak in South Korea. Int J Environ Res Public 2020;17:305.

7. Kim M, Park IH, Kang YS, Kim H, Jhon M, Kim JW, et al. Comparison of psychosocial distress in areas with different COVID-19 prevalence in Korea. Front Psychiatry 2020;11:593105.

8. Spitzer RL, Kroenke K, Williams JB. Validation and utility of a self-report version of PRIME-MD: the PHQ primary care study. Primary Care Evaluation of Mental Disorders. Patient Health Questionnaire. JAMA 1999;282:1737-1744.

9. Spitzer RL, Kroenke K, Williams BW, Löwe B. A brief measure for assessing generalized anxiety disorder: the GAD-7. Arch Intern Med 2006;166:1092-1097.

10. Lee J, Shin C, Ko YH, Lim J, Joe SH, Kim S, et al. The reliability and validity studies of the Korean version of the perceived stress scale. Korean J Psychosom Med 2012;20:127-134.

11. Zarocostas J. How to fight an infodemic. Lancet 2020;395:676.

12. Pollard J. Seen, seared and sealed: trauma and the visual presentation of September 11. Health Risk Soc 2011;13:81-101.

13. Fung IC, Tse ZT, Chan BS, Fu KW. Middle East respiratory syndrome in the Republic of Korea: transparency and communication are key. West Pac Surveill Response J 2015;6:1-2.

14. Collinson S, Khan K, Heffernan JM. The Effects of media reports on disease spread and important public health measurements. PLoS One 2015;10:e0141423.

15. Kinnick KN, Krugman DM. Compassion fatigue: communication and burnout toward social problems. J Mass Commun Q 1996;73:687-707.

16. Sun C, Yang W, Arino J, Khan K. Effect of media-induced social distancing on disease transmission in a two patch setting. Math Biosci 2011;230:87-95.

17. Sliver RC, Holman EA, Andersen JP, Poulin M, Mclntosh DN, Gil-Rivas V. Mental- and physical-health effects of acute exposure to media images of the September 11, 2001, attacks and the Iraq War. Psychol Sci 2013;24:1623-1634.

18. Bishop SJ. Neurocognitive mechanisms of anxiety: an integrative account. Trends Cogn Sci 2007;11:307-316.

19. Aldao A, Nolen-Hoeksema S, Schweizer S. Emotion-regulation strategies across psychopathology: a meta-analytic review. Clin Psychol Rev 2010;30:217-237.

20. Penley JA, Tomaka J, Wiebe JS. The association of coping to physical and psychological health outcomes: a meta-analytic review. J Behav Med 2002;25:551-603.

21. Feldner MT, Zvolensky MJ, Leen-Feldner EW. A critical review of the empirical literature on coping and panic disorder. Clin Psychol Rev 2004;24:123-148.

22. McLean CP, Anderson ER. Brave men and timid women? A review of the gender differences in fear and anxiety. Clin Psychol Rev 2009;29: 
496-505.

23. Li SH, Graham BM. Why are women so vulnerable to anxiety, traumarelated and stress-related disorders? The potential role of sex hormones. Lancet Psychiatry 2017;4:73-82.

24. Liu N, Zhang F, Wei C, Jia Y, Shang Z, Sun L, et al. Prevalence and predictors of PTSS during COVID-19 outbreak in China hardest-hit ar- eas: gender differences matter. Psychiatry Res 2020;287:112921.

25. Wang C, Pan R, Wan X, Tan Y, Xu L, Ho CS, et al. Immediate psychological responses and associated factors during the initial stage of the 2019 coronavirus disease (COVID-19) epidemic among the general population in china. Int J Environ Res Public Health 2020;17:1729. 


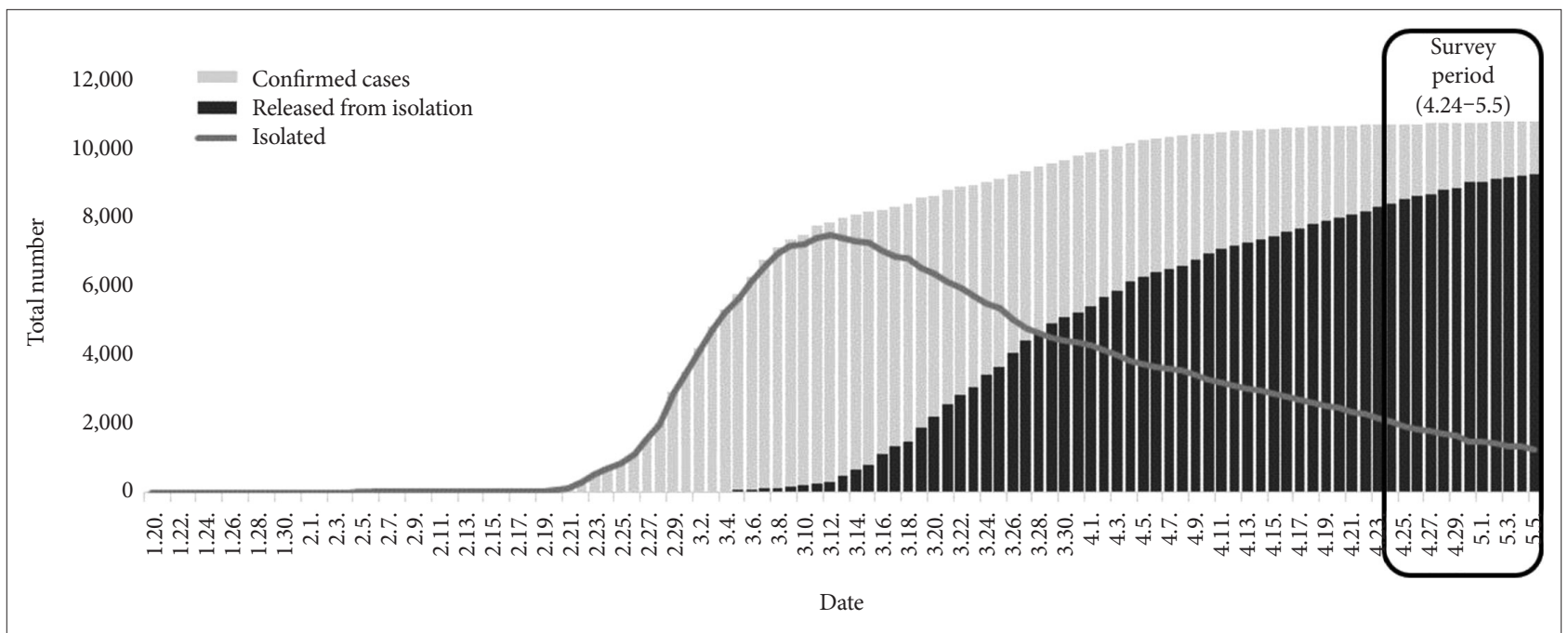

Supplementary Figure 1. The national daily cumulative status of COVID-19 by Korea Centers for Disease Control \& Prevention (N). 